In der Rubrik „Literatur kompakt" werden die wichtigsten Originalarbeiten aus der internationalen Fachliteratur referiert.

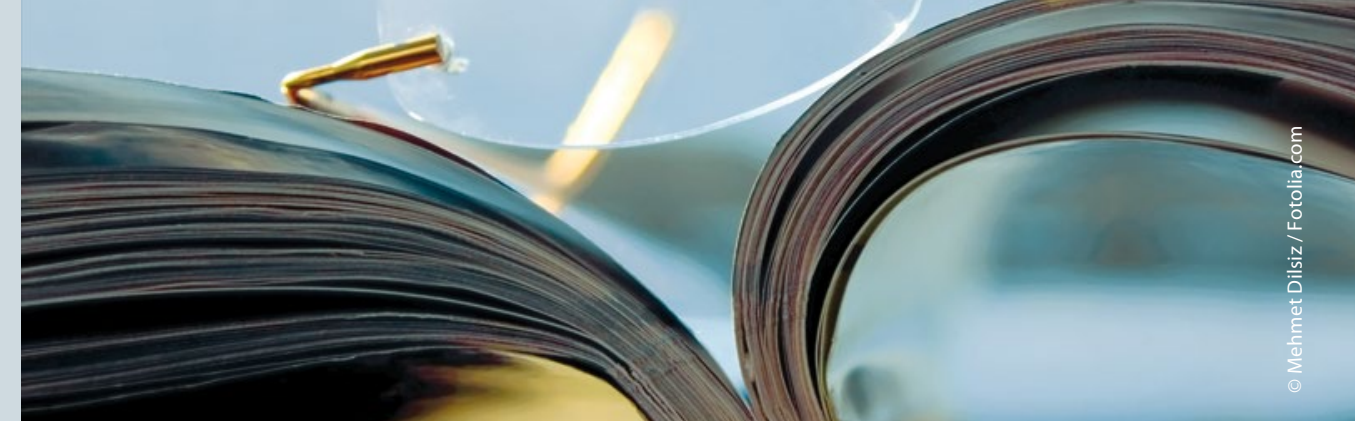

\title{
Probleme mit dem Harnleiterstent: was hilft?
}

\section{Um Probleme durch Harnleiterstents zu vermeiden, sollte auf passende Längen geachtet und der Stent möglichst frühzeitig wieder entfernt werden. Treten dennoch Beschwerden auf, sind laut den Ergebnissen eines Schweizer Reviews wahrscheinlich a-1-Blocker am effektivsten.}

n das systematische Review des Schweizer Autorenteams um Patrick Betschart, St. Gallen, flossen insgesamt 107 Studien zur Prävention und Behandlung stentassoziierter Harnwegsbeschwerden ein.

Wie Betschart et al. berichten, waren Stents, welche die Mittellinie des knöchernen Beckens kreuzen, mit einer erhöhten Rate von Beschwerden assoziiert. Implantate, bei denen das distale Ende unterhalb des Übergangs von der Harnblase in die Harnröhre zu liegen kam, führten in einer randomisierten Studie mit 120 Patienten deutlich häufiger zu Harnwegssymptomen als der Anatomie angepasste, kürzere Stents. Dies schien sich auch auf die Lebensqualität auszuwirken. „Die Mehrzahl der Studien“, so Betschart et al., „scheinen darauf hinzudeuten, dass kürzere Stents, die innerhalb der Harnblase enden, von Vorteil sind".

33 Arbeiten befassten sich mit Material und Design der Ureterstents. Nach Betschart et al. wurden jedoch für keines der getesteten Modelle deutliche Vorteile gefunden. Zwar hätten einzelne Studien nahegelegt, dass weichere Stents mit reduziertem intravesikalem Anteil weniger Irritationen verursachten als konventionelle Modelle. Dagegen schien der Stentdurchmesser keinen wesentlichen Einfluss auf die Symptomatik zu haben. Auch beim Vergleich verschieden beschichteter Stents kam man bislang zu keinem definitiven Ergebnis. Der ideale Stent, so die Autoren, ist offenbar noch nicht entwickelt worden.

Wenig wirksam war das präventive Einbringen verschiedener Substanzen in die Harnblase. In einer Vergleichsstudie reduzierte sich der Symptomscore der Stentpatienten unter Oxybutynin, Lidocain oder Ketorolac nicht mehr als mit physiologischer Kochsalzlösung. Die präventive Injektion mit Botulinumtoxin A führte in einer kleinen randomisierten Studie immerhin zu einer signifikanten Abnahme von Schmerzen und Bedarf an Analgetika. Allerdings verbesserte sich keiner der Parameter im USSQ (Ureteral Stent Symptoms Questionnaire).

Um die Therapie stentbedingter Symptome kommt man demnach in vielen Fällen nicht herum. Nach Betschart und Kollegen haben sich hierfür $\alpha$-1-Blocker als effektiv erwiesen; Tamsulosin offenbar mehr als Alfuzosin. Insbesondere wurden in einer Metaanalyse aus 2016 deutliche Vorteile gefunden, die sich im USSQ-Score niederschlugen. Kein Nutzen zeigte sich im Hinblick auf die Harnspeicherfunktion im IPSS (International Prostate Symptom Score) und

Ein möglicher Zusatzeffekt wird Antimuskarinika attestiert. Dies wird gestützt durch eine Metaanalyse, die 2015 nahelegte, dass die Substanzen in Kombination mit $\alpha-1-B l o c k e r n$ den jeweiliauch nicht in puncto Sexualfunktion. gen Monotherapien überlegen waren. Für sich genommen schienen Antimuskarinika sowohl eine Verbesserung im IPSS-Score als auch eine gesteigerte Lebensqualität zu bewirken. Die Aussagekraft zu Antimuskarinika, so schränken Betschart et al. ein, sei aufgrund erheblicher Heterogenität der Studien jedoch geringer als für die $\alpha$-1-Blocker.

Noch keinerlei belastbares Datenmaterial liegt derzeit für Mirabegron vor. Die Substanz, die seit 2014 zur Behandlung der überaktiven Blase zugelassen ist, wird derzeit in mehreren Studien auf ihre Wirksamkeit bei stentassoziierten Beschwerden getestet. Unklar bleibt bis auf weiteres auch die Rolle klassischer Analgetika. In der klinischen Praxis werden neben Paracetamol auch NSAR, Metamizol oder Opioide eingesetzt. Klinische Studien, die deren Wirksamkeit und Sicherheit bei Stentpatienten prüfen, liegen derzeit nicht vor; dafür besteht laut Betschart et al. durchaus Bedarf.

Fazit: Neben den Ergebnissen ihres Reviews sollte gemäß den Schweizer Experten um Betschart, auch der Aspekt der Patientenaufklärung nicht vernachlässigt werden. In einer eigenen Studie konnten sie nachweisen, dass Patienten, die vor dem Eingriff ausführlich über dessen Ablauf und Folgen informiert worden waren, signifikant weniger Symptome im USSQ berichteten. Abgesehen davon sei es in jedem Fall ratsam, Stents von angemessener Länge zu wählen und diese nicht länger im Harnleiter zu belassen als nötig.

Dr. Elke Oberhofer

Betschart $\mathrm{P}$, et al. Prevention and treatment of symptoms associated with indwelling ureteral stents: a systematic review. Int J Urol. 2017; doi: 10.1111/iju.13311 